\title{
MECANISMO E APLICAÇÕES DA REAÇÃO DE FENTON ASSISTIDA POR COMPOSTOS FENÓLICOS REDUTORES DE FERRO
}

\author{
André Aguiar* e André Ferraz \\ Departamento de Biotecnologia, Escola de Engenharia de Lorena, Universidade de São Paulo, CP 116, 12600-970 Lorena - SP, Brasil \\ David Contreras \\ Departamento de Química Analítica e Inorgânica, Facultad de Ciencias Químicas, Universidade de Concepción, Cp 160-C, \\ Concepción - Chile \\ Jaime Rodríguez \\ Facultad de Ciencias Forestales e Laboratório de Recursos Renováveis, Centro de Biotecnología, Universidade de Concepción, \\ Cp 160-C, Concepción - Chile
}

Recebido em 13/1/06; aceito em 31/5/06; publicado na web em 19/1/07

\begin{abstract}
MECHANISM AND APPLICATIONS OF THE FENTON REACTION ASSISTED BY IRON-REDUCING PHENOLIC COMPOUNDS. The mechanism and applications of the Fenton reaction assisted by iron-reducing phenolic compounds (IRPC) is reviewed. The presence of IRPC leads to the formation of a larger number of free radicals. The relationship between the redox potential and the IRPC structure is discussed. The effect of humic substances in the degradation of xenobiotics is also included, since these substances are able to reduce metallic ions. The natural occurrence of $\mathrm{Fe}^{3+} / \mathrm{H}_{2} \mathrm{O}_{2} / \mathrm{IRPC}$ in wood biodegradation processes, as well as their application is also discussed. The review concludes with the advantages of the $\mathrm{Fe}^{3+} / \mathrm{H}_{2} \mathrm{O}_{2} / \mathrm{IRPC}$ systems and some considerations for further process optimization and their applications at industrial levels.
\end{abstract}

Keywords: Fenton reaction; xenobiotics; iron-reducing phenolic compounds.

\section{OS PROCESSOS OXIDATIVOS AVANÇADOS (POA)}

Xenobióticos são substâncias estranhas aos organismos vivos presentes em águas, solos e na atmosfera, produzidas pela ação do homem e de difícil biodegradabilidade. Para tratamento de águas residuárias contendo compostos não biodegradáveis, os métodos de coagulação química (floculação), adsorção com carvão ativo e separação por membranas demonstram grande eficiência, no entanto, os poluentes retidos (concentrados) necessitam ser posteriormente tratados. Os processos oxidativos avançados (POA) têm recebido grande interesse no tratamento e pré-tratamento de compostos não biodegradáveis em águas, atmosferas e solos contaminados, pois convertem a matéria orgânica em $\mathrm{CO}_{2}$ e $\mathrm{H}_{2} \mathrm{O}$ ou no caso de pré-tratamentos, tornando-os biodegradáveis. Os POA são definidos como processos que geram radicais hidroxila $\left({ }^{\circ} \mathrm{OH}\right) \mathrm{em}$ quantidades suficientes para a degradação de matéria orgânica, empregando diferentes combinações de precursores como $\mathrm{H}_{2} \mathrm{O}_{2}$, $\mathrm{O}_{3}$, luz UV (ultra-violeta), ultra-som e sais de ferro ${ }^{1}$.

Um dos POA mais promissores consiste na reação entre $\mathrm{Fe}^{2+} \mathrm{e}$ $\mathrm{H}_{2} \mathrm{O}_{2}$, conhecida como a reação de Fenton (Equação 1), que gera os radicais $\mathrm{OH}$ que possuem elevado potencial de oxi-redução $(2,3 \mathrm{~V})$, atacando indistintamente todas as espécies presentes no meio reacional $^{2,3}$. A alta reatividade do radical $\mathrm{OH}$ resulta em reações rápidas, da ordem de $1 \times 10^{10}$, e inespecíficas com os distintos substratos, o que implica que a velocidade de reação normalmente é limitada pela velocidade de difusão $0^{4,5}$. Quando é utilizado $\mathrm{Fe}^{3+}$ ao invés de $\mathrm{Fe}^{2+}$ e $\mathrm{H}_{2} \mathrm{O}_{2}$ em excesso (reação tipo Fenton), outros radicais de menor potencial de oxidação como hidroperoxila $\left(\mathrm{HO}_{2}{ }^{\circ}\right)$ e ânion superóxido $\left(\mathrm{O}_{2}^{*}\right)$ também são formados ${ }^{6,7}$. A proporção na qual estes radicais são produzidos é determinada pelo $\mathrm{pH}$, pois a protonação do ânion superóxido ocorre em meio ácido, cujo equilíbrio apresenta pKa 4,8

*e-mail: andrepiranga@yahoo.com.br
$\mathrm{Fe}^{2+}+\mathrm{H}_{2} \mathrm{O}_{2} \rightarrow \mathrm{Fe}^{3+}+{ }^{-} \mathrm{OH}+{ }^{\circ} \mathrm{OH} \quad \mathrm{k}=76 \mathrm{~L} \mathrm{~mol}^{-1} \mathrm{~s}^{-1}, \mathrm{~T}=25^{\circ} \mathrm{C}$ (1, reação de Fenton)

$\mathrm{O}$ radical $\mathrm{OH}$ pode atuar como um eletrófilo ou como um nucleófilo, atacando moléculas orgânicas pela abstração de hidrogênio ou acoplando-se em duplas ligações e anéis aromáticos (hidroxilação), inclusive em posições substituídas causando reações como desmetoxilação $0^{6}$, desalogenação ${ }^{9-11}$, desalquilação ${ }^{12,13}$, desnitração ${ }^{14}$, desaminação ${ }^{15}$ e descarboxilação ${ }^{16,17}$.

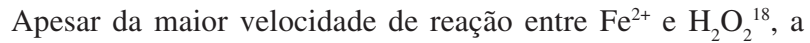
utilização de $\mathrm{Fe}^{3+}$ é mais conveniente, pois neste estado de oxidação o ferro é mais abundante ${ }^{19}$ e tem menor custo. A decomposição de $\mathrm{H}_{2} \mathrm{O}_{2}$ por $\mathrm{Fe}^{3+}$ gera a espécie reduzida $\mathrm{Fe}^{2+}$, que também reage com $\mathrm{H}_{2} \mathrm{O}_{2}$, e o radical hidroperoxila (Equação 2). $\mathrm{O}$ íon $\mathrm{Fe}^{3+}$ também pode ser reduzido por esse radical (Equação 3), gerando radicais $\mathrm{OH}$ pela reação de Fenton.

$$
\begin{aligned}
& \mathrm{Fe}^{3+}+\mathrm{H}_{2} \mathrm{O}_{2} \rightarrow \mathrm{Fe}^{2+}+\mathrm{HO}_{2}^{\cdot}+\mathrm{H}^{+} \mathrm{k}=1 \times 10^{-2} \mathrm{~L} \mathrm{~mol}^{-1} \mathrm{~s}^{-1}, \mathrm{~T}=25^{\circ} \mathrm{C}(2) \\
& \mathrm{Fe}^{3+}+\mathrm{HO}_{2}^{\cdot} \rightarrow \mathrm{Fe}^{2+}+\mathrm{O}_{2}+\mathrm{H}^{+}
\end{aligned}
$$

O cobre também atua como um catalisador na decomposição de $\mathrm{H}_{2} \mathrm{O}_{2}$ (reação de Fenton cuprosa), de modo similar ao ferro. Ambos metais de transição reagem com $\mathrm{H}_{2} \mathrm{O}_{2}$ formando complexos intermediários, que em seguida se decompõem formando o radical $\mathrm{OH}$. Peróxidos orgânicos $\left(\mathrm{RO}_{2} \mathrm{H}\right)$ também são decompostos por esses metais, formando os radicais alcoxila $\left(\mathrm{RO}^{*}\right)$ e peroxila orgânico $\left(\mathrm{RO}_{2}\right)^{2}$. $\mathrm{Na}$ reação de Fenton cuprosa, o complexo formado entre $\mathrm{H}_{2} \mathrm{O}_{2}$ e o metal é mais estável que na reação de Fenton ferrosa ${ }^{20}$. Como consequiência, as espécies ativadas da reação de Fenton cuprosa podem ser o radical $\mathrm{OH}$ ou o íon $\mathrm{Cu}^{3+}$. A reação de Fenton cuprosa é aproximadamente 3 vezes mais rápida que a ferrosa ${ }^{4}$, no entanto, a estabilidade do $\mathrm{Cu}^{1+}$ em solução aquosa depende da presença de quelantes 
ou compostos que reduzam constantemente $\mathrm{Cu}^{2+} \mathrm{a} \mathrm{Cu}^{1+}$.

$\mathrm{Na}$ reação conhecida como foto-Fenton, a luz $\mathrm{UV}$ reduz $\mathrm{Fe}^{3+}$ a $\mathrm{Fe}^{2+}$, o que aumenta a decomposição de $\mathrm{H}_{2} \mathrm{O}_{2}$ e a produção de radicais $\mathrm{OH}$, e fotolisa compostos orgânicos complexados com esse cátion, como oxalato- $\mathrm{Fe}^{3+}$. Alguns sistemas foto-Fenton também podem fazer uso de irradiações de menor energia como as do UV próximo e do visível presentes na luz solar. Nesses casos, a decomposição de $\mathrm{H}_{2} \mathrm{O}_{2}$ ocorre por ocasião do decaimento das espécies ativas geradas nos complexos de $\mathrm{Fe}^{3+1}$. Um outro meio de acelerar a reação entre $\mathrm{Fe}^{3+}$ e $\mathrm{H}_{2} \mathrm{O}_{2}$ para produção de radicais $\mathrm{OH}$ consiste no uso de compostos fenólicos, principalmente os di-hidroxibenzenos (DHB) que apresentam habilidade de reduzir esse metal de transição, que é alvo dessa revisão. A principal vantagem desse sistema é o seu efeito pró-oxidante, devido à maior geração de radicais livres e por um tempo mais prolongado, comparada à reação de Fenton convencional ${ }^{16,17,21-25}$. Os compostos fenólicos regeneram os íons $\mathrm{Fe}^{2+}$ para reagir com $\mathrm{H}_{2} \mathrm{O}_{2}$, possibilitando maior eficiência nos processos de remediação $0^{17,21,22,24}$.

\section{COMPOSTOS FENÓLICOS REDUTORES DE FE ${ }^{3+}$ (CFRF): MECANISMO DE REDUÇÃO E INFLUÊNCIA DE GRUPOS FUNCIONAIS}

Os compostos mais estudados como auxiliares na reação tipo Fenton são os DHB, principalmente os derivados do catecol. A via reacional de redução de $\mathrm{Fe}^{3+}$ pelo catecol está mostrada na Figura 1. O catecol quela o íon $\mathrm{Fe}^{3+}$ formando um complexo que, em seguida, é oxidado monoeletronicamente por um mecanismo de esfera interna (o catecol sendo parte do complexo) gerando um radical semiquinona ${ }^{26}$. Este é posteriormente oxidado monoeletronicamente por um mecanismo de esfera externa (semiquinona não forma parte do complexo) por outro íon, formando uma quinona ${ }^{26,27}$. $\mathrm{O} \mathrm{O}_{2}$ também pode atuar como um aceptor de elétrons, sendo reduzido pelo radical semiquinona aos radicais $\mathrm{O}_{2}-/ \mathrm{HO}_{2}$, que podem ser convertidos em $\mathrm{H}_{2} \mathrm{O}_{2}^{3,19,28-30}$. Um mecanismo semelhante é observado para o íon $\mathrm{Cu}^{2+7,31}$.

Chen et al..$^{17}$ sugeriram que as quinonas podem ser regeneradas aos compostos catecol ou hidroquinona reagindo com o radical $\mathrm{HO}_{2}$ em duas etapas. Chen e Pignatello ${ }^{32}$ observaram que na presença de luz UV, uma quinona em solução é regenerada ao radical semiquinona com a geração simultânea de radical $\mathrm{OH}$, uma outra rota de produção desse forte agente oxidante.

A atividade oxidante de íons $\mathrm{Fe}^{3+}$ e $\mathrm{Cu}^{2+}$ tem sido avaliada para vários compostos fenólicos ${ }^{7,21,22,33-36}$. Pracht et $a l .{ }^{34}$ observaram que catecol, guaiacol e hidroquinona, exceto resorcinol reduziram $\mathrm{Fe}^{3+}$ em sua forma solúvel e insolúvel. Catecol e guaiacol reduziram o íon $\mathrm{Fe}^{3+}$ em uma relação molar acima de 2:1 e foram mineralizados. A oxidação das $o$-quinonas formadas por $\mathrm{Fe}^{3+}$ foi sugerida, enquanto a hidroquinona reduziu $\mathrm{Fe}^{3+}$ na relação 2:1 sem sofrer mineralização.

Aguiar e Ferraz ${ }^{35}$ avaliaram a redução de íons $\mathrm{Fe}^{3+} \mathrm{e} \mathrm{Cu}^{2+} \mathrm{em} \mathrm{pH}$ 4,5 por cerca de 20 compostos do tipo catecol, resorcinol, hidroquinona, pirogalol, guaiacol e siringol. As principais estruturas dos compostos fenólicos avaliados são mostradas na Figura 2. Para os DHB, as posições das hidroxilas no anel aromático exercem forte influência na redução dos íons metálicos. Derivados do catecol, pirogalol e hidroquinona, principalmente os ácidos 3,4-di-hidroxi-fenilacético, gálico (3,4,5-tri-hidroxi-benzóico) e 2,5-di-hidroxi-tereftálico foram os mais eficientes na redução de $\mathrm{Fe}^{3+}$ e $\mathrm{Cu}^{2+}$. A redução inesperada de $\mathrm{Fe}^{3+}$ por derivados do resorcinol (ácidos 2,4- e 2,6-di-hidroxibenzóico) foi observada, mas não para o cobre. Pracht et al ${ }^{34}$ relataram que resorcinol não reduz $\mathrm{Fe}^{3+}$, pois não pode ser convertido em quinonas. No entanto, os possíveis radicais semiquinona formados pela oxidação desse composto poderiam se acoplar, formando dímeros ou outros produtos de oxidação. A maioria dos compostos avaliados reduziu mais o íon $\mathrm{Fe}^{3+}$ que $\mathrm{Cu}^{2+}$ e acima da relação 2:1. Foi também observado que outros grupos funcionais $\left(-\mathrm{CO}_{2} \mathrm{H},-\mathrm{CHO},-\mathrm{CH}_{2} \mathrm{OH}\right.$, $-\mathrm{CH}=\mathrm{CH}-\mathrm{CO}_{2} \mathrm{H}$ ) presentes no anel aromático de vários compostos afetaram diferentemente o comportamento dessas reações. Entre os derivados do guaiacol, a vanilina (3-metoxi-4-hidroxi-benzaldeído) foi menos eficiente na redução de $\mathrm{Fe}^{3+}$ que os ácidos vanílico (3metoxi-4-hidroxi-benzóico) e ferúlico (3-metoxi-4-hidroxicinâmico). O álcool vanílico (3-metoxi-4-hidroxi-benzílico) foi o mais eficiente na redução de $\mathrm{Fe}^{3+}$, enquanto a vanilina foi o único derivado do guaiacol que não reduziu $\mathrm{Cu}^{2+}{ }^{35}$.

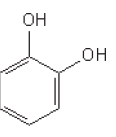

(1)

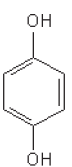

(2)

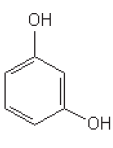

(3)

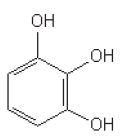

(4)

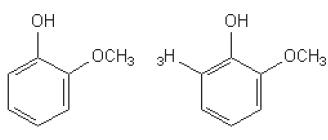

(5)
(6)
Figura 2. Estruturas dos compostos fenólicos avaliados na redução de $\mathrm{Fe}^{3+}$ e $\mathrm{Cu}^{2+}:$ 1-catecol, 2- hidroquinona, 3-resorcinol, 4-pirogalol, 5- guaiacol e 6- siringol ${ }^{35}$

\section{APLICAÇ̃̃o DE CFRF NA DEGRADAÇÃo DE XENOBIÓTICOS PELA REAÇÃO DE FENTON}

O primeiro trabalho que descreveu a degradação de xenobióticos pela reação de Fenton assistida pelo catecol foi desenvolvido por Hamilton et $a l .^{37}$, o que posteriormente foi denominado de sistema de Hamilton. Foram estudadas reações de hidroxilação do benzeno, cloro-benzeno, nitro-benzeno e anisol (metoxi-benzeno) por $\mathrm{Fe}^{3+} \mathrm{e}$ $\mathrm{H}_{2} \mathrm{O}_{2}$ na presença de catecol, hidroquinona, ácido cloranílico e $p$ quinona. Na Tabela 1 são mostrados vários trabalhos que empregaram diferentes CFRF em reações do tipo Fenton para oxidação de xenobióticos.

Alguns compostos analisados por Sun e Pignatello ${ }^{12}$ que mediaram a reação entre $\mathrm{Fe}^{3+} \mathrm{e}_{2} \mathrm{O}_{2}$ apresentaram um período inicial de indução para degradar o ácido 2,4-dicloro-fenoxi-acético. Uma solução envelhecida $(4 \mathrm{~h})$ contendo o flavonóide quercetina, violeta de pirocatecol ou o próprio catecol com $\mathrm{Fe}^{3+} \mathrm{e}_{2} \mathrm{O}_{2}$ foi mais rápida e eficiente na degradação do pesticida, mostrando que os produtos intermediários formados entre os CFRF, $\mathrm{Fe}^{3+}$ e $\mathrm{H}_{2} \mathrm{O}_{2}$ são agentes oxidantes mais fortes que seus precursores. A concentração de CFRF nessas reações é um parâmetro muito importante que deve ser otimizado. O aumento da concentração de ácido picolínico (2carboxi-piridina) na oxidação do ácido 2,4-dicloro-fenoxi-acético

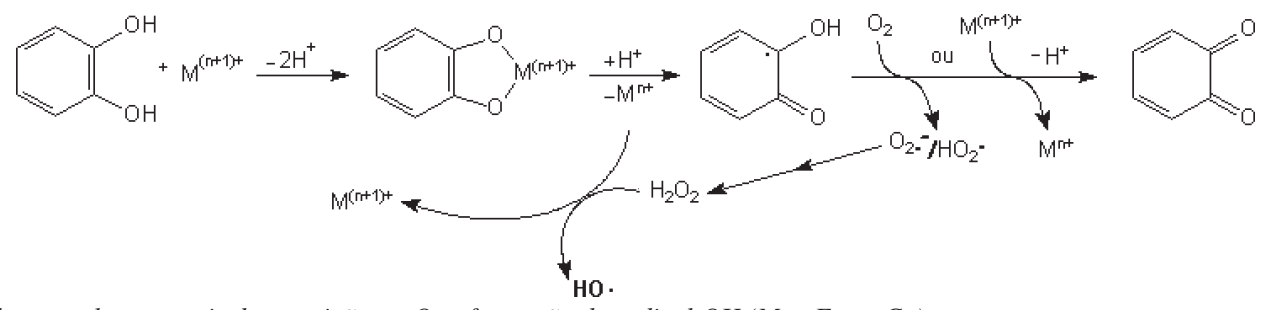

Figura 1. Oxidação de catecol por metais de transição ou $\mathrm{O}_{2}$ e formação de radical $\mathrm{OH}(\mathrm{M}=\mathrm{Fe}$ ou $\mathrm{Cu})$ 
Tabela 1. Compostos xenobióticos degradados por $\mathrm{Fe}^{3+}$ ou $\mathrm{Fe}^{2+}$ e $\mathrm{H}_{2} \mathrm{O}_{2}$ na presença de compostos fenólicos

\begin{tabular}{|c|c|c|c|}
\hline Xenobióticos & Tratamento & Compostos fenólicos & Ref. \\
\hline ácido 2,4-dicloro-fenoxi-acético & $\mathrm{Fe}^{3+} / \mathrm{H}_{2} \mathrm{O}_{2}$ & $\begin{array}{l}\text { ácidos gálico, rodizônico, picolínico* e } \\
\text { 2,3-DHB, catecol, quercetina, } \\
\text { tetra-1,4-hidroxi-quinona, violeta de pirocatecol }\end{array}$ & 12 \\
\hline pesticidas & $\mathrm{Fe}^{3+} / \mathrm{H}_{2} \mathrm{O}_{2}$ & ácidos gálico, rodizônico e picolínico & 13 \\
\hline ácido 2,4-dicloro-fenoxi-acético & $\begin{array}{l}\mathrm{Fe}^{3+} / \mathrm{H}_{2} \mathrm{O}_{2} / \mathrm{luz} \mathrm{UV} \\
(300-400 \mathrm{~nm})\end{array}$ & ácidos gálico, rodizônico e picolínico & 13 \\
\hline verde malaquita & $\mathrm{Fe}^{3+} / \mathrm{H}_{2} \mathrm{O}_{2}$ & $\begin{array}{l}\text { catecol, hidroquinona, resorcinol, ácidos salicílico, } \\
p \text { - e } m \text {-hidroxi-benzóico e ftálico*, } p \text {-quinona* }\end{array}$ & 17 \\
\hline $\begin{array}{l}\text { 2- e 4-cloro-fenóis, efluente } \\
\text { kraft, álcool veratrílico }\end{array}$ & $\mathrm{Fe}^{3+}\left(\mathrm{Fe}^{2+}\right) / \mathrm{H}_{2} \mathrm{O}_{2}$ & ácidos 2,3-DHB e protocatecóico, catecol & 21,22 \\
\hline corantes & $\mathrm{Fe}^{2+} / \mathrm{H}_{2} \mathrm{O}_{2}$ & ácido 2,3-DHB & 24 \\
\hline $\begin{array}{l}\text { benzeno, cloro-benzeno, } \\
\text { nitro-benzeno, anisol }\end{array}$ & $\mathrm{Fe}^{3+} / \mathrm{H}_{2}^{2} \mathrm{O}_{2}^{2}$ & catecol, hidroquinona, ácido cloranílico*, $p$-quinona & 37 \\
\hline HAP & $\mathrm{Fe}^{3+} / \mathrm{H}_{2} \mathrm{O}_{2}$ & ácido gálico, catecol & 38 \\
\hline verde malaquita & $\mathrm{Fe}^{3+} / \mathrm{H}_{2} \mathrm{O}_{2} /$ luz visível & $\begin{array}{l}\text { catecol, hidroquinona, resorcinol, } \\
\text { ácidos salicílico e ftálico, } p \text {-quinona }\end{array}$ & 39 \\
\hline $\mathrm{EDTA}^{-\mathrm{Fe}^{3+}}$ & $\mathrm{H}_{2} \mathrm{O}_{2}$ & catecol & 40,41 \\
\hline
\end{tabular}

*compostos não fenólicos

causou efeito inibitório, possivelmente devido ao efeito de competição entre esses dois compostos pelos radicais $\mathrm{OH}$. Chen et al. ${ }^{17}$ também observaram inibição na degradação dos corantes verde malaquita e rodamina $6 \mathrm{G}$ com o aumento da concentração de ácido salicílico. Apesar deste composto e também dos ácidos $p$ - e $m$ hidroxi-benzóico, ftálico e o resorcinol não reduzirem $\mathrm{Fe}^{3+}$, foi observado que o ácido salicílico sofreu reações de hidroxilação levando à formação de derivados do catecol e da hidroquinona, dessa forma acelerando a degradação dos corantes.

Sun e Pignatello ${ }^{13}$ não observaram uma ordem de reatividade entre os CFRF e os substratos (pesticidas) estudados. Por ex., a degradação do ácido 2,4,5-tricloro-fenoxi-acético por $\mathrm{Fe}^{3+}$ e $\mathrm{H}_{2} \mathrm{O}_{2}$ foi mais eficiente na presença do ácido rodizônico, enquanto na presença dos ácidos picolínico ou gálico houve maior mineralização deste pesticida. Por outro lado, atrazina foi completamente degradada na presença dos ácidos gálico, rodizônico e picolínico após 30, 60 e $90 \mathrm{~min}$, respectivamente, sem sofrer mineralização. O efeito positivo de CFRF tem sido atribuído à mais alta e duradoura formação de radicais $\mathrm{OH}$ e radicais livres totais ${ }^{16,21-25}$. Alguns estudos também têm avaliado a eficiência de CFRF com o metal em seu estado reduzido no início das reações. Mesmo nesse sistema $\left(\mathrm{Fe}^{2+} / \mathrm{H}_{2} \mathrm{O}_{2} /\right.$ CFRF) a participação de fenóis estimula a formação de radicais livres e a oxidação de xenobióticos ${ }^{22,24}$.

Um exemplo de degradação de composto alifático pelo sistema $\mathrm{Fe}^{3+} / \mathrm{H}_{2} \mathrm{O}_{2} / \mathrm{CFRF}$ foi relatado por Oviedo et al. ${ }^{40}$. Nesse caso, $40 \%$ de EDTA (ácido etileno-diamino-tetra-acético)- $\mathrm{Fe}^{3+}$ presente em solução foi degradado em $2 \mathrm{~h}$ de tratamento por um sistema que continha $2 \times 10^{-3} \mathrm{~mol} \mathrm{~L}^{-1}$ de $\mathrm{H}_{2} \mathrm{O}_{2}$ e $5 \times 10^{-5} \mathrm{~mol} \mathrm{~L}^{-1}$ de catecol. Com um aumento de $2 \times 10^{-3}$ para $2 \times 10^{-2} \mathrm{~mol} \mathrm{~L}^{-1}$ de $\mathrm{H}_{2} \mathrm{O}_{2}, 90 \%$ de EDTA-Fe ${ }^{3+}$ foi degradado com $20 \%$ de perda de carbono orgânico total (COT).

$\mathrm{O}$ pH do meio é um parâmetro muito importante em reações do tipo Fenton, pois o $\mathrm{Fe}^{3+}$ é solúvel em água somente em valores de pH abaixo de 2 a $3^{30}$. Entretanto, a formação de complexos estáveis entre os CFRF e Fe ${ }^{3+}$ em valores de $\mathrm{pH}$ entre 5 e 6,5 tem sido obser$\operatorname{vada}^{12,13,38,42}$, dispensando a acidificação de amostras a serem tratadas, principalmente no caso de solos e efluentes alcalinos.

A integração entre tratamento biológico e sistema $\mathrm{Fe}^{3+} / \mathrm{H}_{2} \mathrm{O}_{2} /$ CFRF também tem proporcionado resultados satisfatórios. Nam et $a l .{ }^{38}$ avaliaram a degradação de hidrocarbonetos aromáticos policíclicos (HAP) extraídos de uma amostra de solo por $\mathrm{Fe}^{3+}$ e $\mathrm{H}_{2} \mathrm{O}_{2}$ em pH 6,0-6,5, empregando CFRF (catecol ou ácido gálico).
O tratamento químico ( $24 \mathrm{~h}$ ) prévio ao tratamento com bactérias (4 semanas) proporcionou mais de $98 \%$ de degradação dos HAP de 2 e 3 anéis (naftaleno, fluoreno, fenantreno, antraceno) e entre 70 e 85\% dos HAP de 4 e 5 anéis (pireno, criseno, benzo[a]pireno). O tratamento biológico prévio ao tratamento químico foi menos eficiente. Oviedo et $a l .{ }^{41}$ demonstraram que a oxidação química com $\mathrm{H}_{2} \mathrm{O}_{2}$ e catecol $(6 \mathrm{~h})$ prévia ao tratamento biológico com a levedura Saccharomyces cerevisiae ( 9 dias) promoveu $100 \%$ de degradação

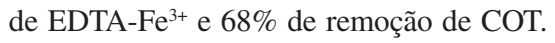

Um método diferente de avaliar a ação de aditivos fenólicos na reação de Fenton foi realizado por Mai et $a l .^{43}$. A incorporação de lignossulfonato, guaiacol ou ácido protocatecóico em polímeros de acrilamida e ácido acrílico foi efetuada para acelerar a degradação destes copolímeros pela reação de Fenton. Os copolímeros de acrilamida contendo lignossulfonato ou guaiacol foram mais rapidamente degradados. Esse efeito foi atribuído à reação mais rápida entre esses copolímeros com os radicais $\mathrm{OH}$. Todos os três copolímeros de ácido acrílico apresentaram maior redução da massa molar média em relação ao homopolímero (controle). Os copolímeros de guaiacol apresentaram maior redução da massa molar e apresentaram o menor consumo de $\mathrm{H}_{2} \mathrm{O}_{2}$. Entretanto, neste estudo não foi comentada a regeneração dos íons $\mathrm{Fe}^{2+}$ pelos compostos que foram incorporados.

Em POA, a formação de CFRF a partir de xenobióticos tem sido observada em uma série de tratamentos ${ }^{37,39}$, inclusive na presença de substâncias húmicas ${ }^{10,15}$ e em processos de biorremediação $0^{9,11}$. Certas amostras de efluentes, como das indústrias de celulose e papel, possuem grandes quantidades de compostos aromáticos provenientes da degradação de lignina e extrativos que apresentam estruturas do tipo catecol, pirogalol, guaiacol e siringol ${ }^{44}$, podendo também facilitar a degradação da matéria orgânica presente.

Estudos realizados com compostos não aromáticos e que aparentemente não são agentes redutores de $\mathrm{Fe}^{3+}$ ou $\mathrm{Cu}^{2+}$, exceto o ácido ascórbico ${ }^{16}$, também têm estimulado a degradação de xenobióticos por $\mathrm{H}_{2} \mathrm{O}_{2}$ e um metal de transição na faixa de $\mathrm{pH}$ entre 3 e 9. Os ácidos succínico, glucárico e ascórbico, piridina e d-arabinono-1,4lactona têm sido avaliados na formação de complexos com cátions metálicos, principalmente $\mathrm{Cu}^{2+}$ ou $\mathrm{Co}^{2+}$, na degradação de corantes, HAP, benzeno e derivados ${ }^{45}$. Apesar do uso de cobre ou cobalto nesses sistemas apresentar grande eficiência, a toxicidade desses metais pode causar outros problemas ambientais. 


\section{EFEITO DE SUBSTÂNCIAS HÚMICAS EM POA}

Na natureza, os microorganismos consomem fontes de carbono mais facilmente assimiláveis como monossacarídeos, amido, proteínas e lipídios. Moléculas mais resistentes ao ataque microbiológico como celulose, polioses e lignina são parcialmente consumidas, mineralizadas ou então transformadas em húmus. Substâncias húmicas podem ser consideradas como o maior reservatório de carbono orgânico em águas e nos solos. A estrutura definida destas substâncias é desconhecida, mas são divididas em grupos de acordo com a solubilidade em água e o pH. Humina corresponde à fração insolúvel e os ácidos fúlvicos à fração solúvel em água, independente do $\mathrm{pH}$. Os ácidos húmicos são solúveis apenas em $\mathrm{pH}$ alcalino $^{46}$. Na Figura 3 são mostradas partes estruturais propostas para substâncias húmicas.

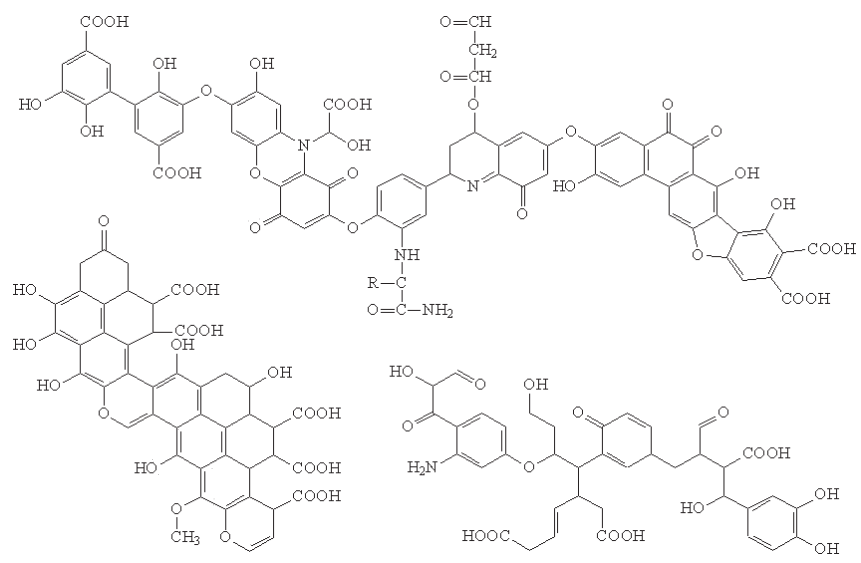

Figura 3. Estruturas parciais de ácidos húmicos. Adaptadas da ref. 47

Em muitos estudos de degradação de compostos xenobióticos por POA, faz-se necessária a realização dos ensaios na presença de matéria orgânica natural, com o intuito de simular e entender os processos de remediação no meio ambiente. De acordo com Voelker e Sulzberger ${ }^{48}$, várias reações simultâneas ocorrem entre $\mathrm{Fe}^{2+}, \mathrm{H}_{2} \mathrm{O}_{2}$, $\mathrm{O}_{2}$ e ácidos fúlvicos em solução. Nesse trabalho foi observado que os complexos fulvato- $\mathrm{Fe}^{2+}$ foram mais eficientes que os complexos aquo- $\mathrm{Fe}^{2+}$ na decomposição de $\mathrm{H}_{2} \mathrm{O}_{2} \mathrm{em}$ pH 5. Em pH 3, esse efeito foi desprezível. Os radicais $\mathrm{OH}$ gerados no meio foram seqüestrados pelos ácidos fúlvicos, reduzindo $\mathrm{O}_{2}$ molecular a $\mathrm{O}_{2}{ }^{*} / \mathrm{HO}_{2}{ }^{*}$ regenerando $\mathrm{H}_{2} \mathrm{O}_{2}$. Foi observado também que os ácidos fúlvicos reduzem mais $\mathrm{Fe}^{3+}$ com a diminuição do $\mathrm{pH}$. Paciolla et al. ${ }^{47}$ observaram que ácidos húmicos podem quelar íons $\mathrm{Fe}^{3+}$ e $\mathrm{Cu}^{2+} \mathrm{e}$ os complexos formados podem gerar radicais $\mathrm{OH}$ ao reagir com $\mathrm{H}_{2} \mathrm{O}_{2}$. Lindsey e Tarr $^{49}$ mostraram que um aumento de $1 \times 10^{-2}$ para $3 \times 10^{-2} \mathrm{~g} \mathrm{~L}^{-1} \mathrm{de}$ ácidos húmicos reduziu a formação de radicais $\mathrm{OH}$ por $\mathrm{Fe}^{2+} \mathrm{e}_{2} \mathrm{O}_{2}$ em $\mathrm{pH}$ próximo de 3 , enquanto que em meio reacional levemente ácido (pH entre 4,5 e 6,5) os ácidos fúlvicos estimularam a reação. Estes mesmos autores ao comparar diferentes substâncias húmicas, observaram que $3 \times 10^{-2} \mathrm{~g} \mathrm{~L}^{-1}$ de ácidos húmicos diminuiu em $27 \%$ a formação de radicais $\mathrm{OH}$, enquanto a mesma concentração de ácidos fúlvicos inibiu apenas $11 \%$. De acordo com esses e outros trabalhos, substâncias húmicas devem interferir na degradação de xenobióticos por POA, seja quelando e/ou reduzindo íons metáli$\cos ^{47,48}$, seqüestrando os radicais livres ou estimulando a produção destes para degradação de xenobióticos ${ }^{10,15,47-49}$, ou ainda incorporando os produtos de oxidação de xenobióticos em suas estruturas poliméricas $^{10,15}$.

A Tabela 2 compila alguns trabalhos que avaliaram a participação de substâncias húmicas na degradação de xenobióticos por reações do tipo Fenton. Uma análise ampla desses trabalhos indica que seriam necessários estudos correlacionando o efeito dessas substâncias de acordo com a presença e conteúdo de grupos funcionais que apresentam forte atividade quelante e redutora de íons metálicos.

\section{OCORRÊNCIA DO SISTEMA $\mathrm{Fe}^{3+} / \mathrm{H}_{2} \mathrm{O}_{2} / \mathrm{CFRF}$ NA BIODEGRADAÇÃO DE MADEIRA E SUA APLICAÇÃO EM BIORREMEDIAÇÃO}

A madeira é composta principalmente de celulose, polioses e lignina e uma fração menor que corresponde aos extrativos. Os principais organismos que decompõem esse material complexo são os fungos de decomposição parda e branca. Os fungos de decomposição branca degradam todos os componentes da madeira, embora algumas espécies ataquem seletivamente a lignina em estágios iniciais de colonização $0^{53}$. Os fungos de decomposição parda degradam preferencialmente os polissacarídeos ${ }^{53}$, entretanto, muitas espécies carecem de um sistema enzimático hidrolítico completo ${ }^{19}$. Koenigs ${ }^{54}$ propôs que esses fungos podem degradar os polissacarídeos por um sistema não enzimático, envolvendo o ferro presente na madeira e $\mathrm{H}_{2} \mathrm{O}_{2}$ produzido pelo próprio organismo na geração de radicais $\mathrm{OH}$ via reação de Fenton. Como esse metal é encontrado predominantemente na madeira em sua forma oxidada ${ }^{19}$, a produção de agentes quelantes para solubilizá-lo e reduzi-lo é de extrema importância, como o ácido oxálico, produzido por fungos de decomposição parda e branca. No entanto, esse quelante necessita de luz para reduzir $\mathrm{Fe}^{3+19,55}$. Goodell et al. ${ }^{19}$ mostraram que um extrato de baixa massa molar (menor que $1 \mathrm{~kg} \mathrm{~mol}^{-1}$ ) de um cultivo do fungo Gloeophyllum trabeum possuía compostos do tipo catecol com atividade redutora de $\mathrm{Fe}^{3+}$. Esses compostos exibiram atividade pró-oxidante, devido à maior formação de radicais $\mathrm{OH}$ entre o metal e $\mathrm{H}_{2} \mathrm{O}_{2}$. Esses estudos permitiram concluir que esse fungo utiliza um ciclo extracelular de oxi-redução de quinonas para gerar os dois reagentes de Fenton. Anos depois, Kerem et al. ${ }^{28}$ e Paszczynski et al..$^{56}$ identificaram dois agentes redutores em cultivos desse mesmo fungo: 2,5-dimetoxihidroquinona (2,5-DMHQ) e 4,5-dimetoxi-catecol (4,5-DMC) e suas respectivas formas oxidadas. Na presença de micélio fúngico, 2,5-

Tabela 2. Compostos xenobióticos degradados por reações do tipo Fenton na presença de substâncias húmicas

\begin{tabular}{|c|c|c|c|c|}
\hline Xenobiótico & Efeito & Substâncias húmicas & Tratamento & Ref. \\
\hline PCF & estímulo & $5 \times 10^{-2} \mathrm{~g} \mathrm{~L}^{-1}$ de ácidos húmicos & $\mathrm{Fe}^{3+} / \mathrm{H}_{2} \mathrm{O}_{2} /$ luz visível & 10 \\
\hline TNT* & inibição & ácidos húmicos $\left(1,2\right.$ e 4 x $10^{-2} \mathrm{~g}$ de carbono $\left.\mathrm{L}^{-1}\right)$ & $\mathrm{Fe}^{2+} / \mathrm{H}_{2} \mathrm{O}_{2}$ & 14 \\
\hline TNT & estímulo & ácidos fúlvicos $\left(1,2\right.$ e $4 \times 10^{-2} \mathrm{~g}$ de carbono $\left.\mathrm{L}^{-1}\right)$ & $\mathrm{Fe}^{2+} / \mathrm{H}_{2} \mathrm{O}_{2}, \mathrm{Fe}^{2+} / \mathrm{H}_{2} \mathrm{O}_{2} / \mathrm{luz} \mathrm{UV}$ & 14 \\
\hline anilina & estímulo & 2,5 e $10 \times 10^{-2} \mathrm{~g} \mathrm{~L}^{-1}$ de ácidos húmicos & $\mathrm{Fe}^{3+} / \mathrm{H}_{2} \mathrm{O}_{2} /$ luz visível & 15 \\
\hline fenol e HAP & inibição & $5,10,30 \times 10^{-3} \mathrm{~g} \mathrm{~L}^{-1}$ de ácidos fúlvicos & $\mathrm{Fe}^{2+} / \mathrm{H}_{2} \mathrm{O}_{2}$ & 50 \\
\hline fluoreno & inibição & $2,5 \times 10^{-2} \mathrm{~g} \mathrm{~L}^{-1}$ de substâncias húmicas & $\mathrm{Fe}^{2+} / \mathrm{H}_{2}^{2} \mathrm{O}_{2}^{2}$ & 51 \\
\hline fenol & estímulo & 0,5 a $8 \times 10^{-2} \mathrm{~g} \mathrm{~L}^{-1}$ de ácidos húmicos & $\mathrm{Fe}^{3+} / \mathrm{H}_{2} \mathrm{O}_{2}$ & 52 \\
\hline
\end{tabular}

*trinitro-tolueno 
dimetoxi-benzoquinona (2,5-DMBQ) foi capaz de reduzir $\mathrm{Fe}^{3+}$ a $\mathrm{Fe}^{2+}$ que, na presença de $\mathrm{H}_{2} \mathrm{O}_{2}$ produzido pelo fungo, degradou polietileno-glicol (PEG) como substrato modelo de celulose. Na ausência de ferro, não houve degradação do polímero ${ }^{28}$. O composto 2,5-DMBQ não reduziu $\mathrm{Fe}^{3+}$ diretamente, mas sim na presença de micélio fúngico, que possui uma enzima que catalisa a conversão de quinonas em suas respectivas formas reduzidas ${ }^{57}$. A reação entre 2,5-DMHQ e $\mathrm{Fe}^{3+}$ gerou $\mathrm{H}_{2} \mathrm{O}_{2}$, promovendo a degradação de PEG mesmo na ausência do micélio ${ }^{28}$.

Alguns estudos têm utilizado os metabólitos produzidos pelo fungo G. trabeum como pró-oxidantes na degradação de xenobióticos ${ }^{19,58}$ e estes tratamentos têm sido denominados de processos oxidativos avançados fúngicos (POAF ${ }^{22}$. Um aumento de $30 \%$ na degradação de pentacloro-fenol (PCF) nas reações entre íons ferro $\left(\mathrm{Fe}^{2+}\right.$ ou $\left.\mathrm{Fe}^{3+}\right)$ e $\mathrm{H}_{2} \mathrm{O}_{2}$ mediada pelos metabólitos de $G$. trabeum foi observado ${ }^{19}$. Kramer et al. ${ }^{11}$ avaliaram a degradação de 2-flúor-fenol dependente de $\mathrm{Fe}^{2+}$ ou $\mathrm{Fe}^{3+}$ em culturas líquidas do fungo G. striatum. Em uma cultura de 2 dias, sem adição de metal e 2-flúor-fenol, foi detectada atividade redutora de $\mathrm{Fe}^{3+}$ e a presença de 2,5-DMHQ e 4,5-DMC. A degradação de 2,4-dicloro-fenol em culturas desse mesmo fungo foi também avaliada. A adição de manitol (agente seqüestraste de radicais livres) ou ausência de íons ferro nos cultivos inibiu a formação de radical $\mathrm{OH}$ e também a mineralização do organoclorado?.

Enoki et al.$^{59}$ revisaram a produção de glicopeptídeos de baixa massa molar ligados ao íon $\mathrm{Fe}^{2+}$ por fungos de decomposição branca e parda. Supõe-se que esses compostos realizam reações de oxiredução entre $\mathrm{O}_{2}$ e um doador de elétrons, como o NADH (nicotinamida-adenina-dinucleotídeo reduzido), gerando radicais $\mathrm{OH}$ via reação de Fenton. Porém, nenhum estudo tem avaliado a ação desses metabólitos fúngicos em biorremediação.

\section{OUTRAS APLICAÇÕES DO SISTEMA $\mathrm{Fe}^{3+} / \mathrm{H}_{2} \mathrm{O}_{2} / \mathrm{CFRF}$}

Além da degradação de xenobióticos, a reação de Fenton assistida por CFRF também tem sido estudada em reciclagem de papel, deslignificação de polpas e na formação de radicais fenoxila na superfície de fibras de polpas para obtenção de painéis ("fiberboards").

Qian e Goodell ${ }^{60}$ avaliaram três processos para remoção de tinta de impressão a laser em folhas de papel: descoloração alcalina com $\mathrm{NaOH}$ (método convencional); descoloração oxidativa com os reagentes Fenton mais os ácidos 2,3-DHB (2,3-di-hidroxi-benzóico) e oxálico e, descoloração alcalina seguida de tratamento oxidativo. Após esses tratamentos não foi observada nenhuma diferença, mas seguido de uma etapa de flotação, o processo oxidativo apresentou maior remoção de tinta comparado ao tratamento alcalino. A combinação dos dois processos foi ainda mais eficiente, sendo atribuído à maior acessibilidade dos radicais livres às cadeias de celulose ligadas à tinta pelo pré-tratamento alcalino. Por meio de medidas de drenabilidade, comprimento das fibras, índices de resistência à tração e ao rasgo das polpas não foi constatada nenhuma perda de qualidade pelos tratamentos oxidativos. A alvura da polpa tratada com $\mathrm{NaOH}$ foi semelhante ao papel original (controle, sem impressão), enquanto os tratamentos oxidativos apresentaram redução dessa propriedade devido à formação de complexos coloridos entre o ácido 2,3-DHB e os íons ferro ${ }^{12,33}$.

Apesar do trabalho promissor citado anteriormente, um estudo aplicado na deslignificação de polpas mostrou resultados insatisfatórios. Parra et $a l .{ }^{61}$ avaliaram a deslignificação de polpa kraft de Pinus radiata na presença de catecol ou ácido 2,3-DHB. A deslignificação foi avaliada por meio de medidas do número Kappa, que representa o teor de lignina e os produtos de degradação da madeira retidos na polpa. Na sequiência de branqueamento CFRF/ extração alcalina/ $\mathrm{H}_{2} \mathrm{O}_{2}$ houve redução do número Kappa e da viscosidade das polpas, não havendo seletividade. Ao adicionar $\mathrm{H}_{2} \mathrm{O}_{2}$ na primeira etapa, as reduções de viscosidade e do número Kappa foram mais expressivas ainda, pois o ferro presente nas polpas pode ter reagido com $\mathrm{H}_{2} \mathrm{O}_{2}$, que na presença de CFRF favoreceu ainda mais a formação de radicais $\mathrm{OH}$.

Em um estudo de ativação de fibras de polpa termomecânica para a produção de "fiberboards", Qian et al. ${ }^{24}$ avaliaram a geração de radicais fenoxila como um mecanismo de auto-adesão da lignina e extrativos na superfície das fibras. $\mathrm{O}$ tratamento com reagentes de Fenton assistido pelo ácido 2,3-DHB promoveu maior formação de radicais $\mathrm{OH}$ em solução (sem fibras) e por um tempo maior (15 min) que o sistema Fenton convencional (1 min). Porém, na ausência do CFRF, foi observada maior formação de radicais fenoxila nas fibras. Os autores sugeriram que a formação excessiva de radicais $\mathrm{OH}$ degrada os radicais fenoxila. De fato, altas concentrações de reagentes de Fenton na presença de CFRF $\left(1 \times 10^{-3} \mathrm{~mol} \mathrm{~L}^{-1}\right.$ de $\mathrm{FeCl}_{2}$, $5 \times 10^{-3} \mathrm{~mol} \mathrm{~L}^{-1}$ de $\mathrm{H}_{2} \mathrm{O}_{2}$ e $1 \times 10^{-3} \mathrm{~mol} \mathrm{~L}^{-1}$ de ácido 2,3-DHB), diminuíram a formação de radicais nas fibras, enquanto que em concentrações 10 vezes menores, a formação foi favorecida. Qian $e t$ $a l .{ }^{25}$ apresentaram dados adicionais do estudo anteriormente descrito utilizando também o cobre. A formação de radicais $\mathrm{OH}$ por $\mathrm{Cu}^{2+}$ correspondeu a apenas $6 \%$ do valor determinado para $\mathrm{Fe}^{2+}$. A adição do ácido 2,3-DHB na reação entre $\mathrm{Cu}^{2+}$ e $\mathrm{H}_{2} \mathrm{O}_{2}$ exerceu efeito inibitório.

Yelle et al. ${ }^{62}$ avaliaram as propriedades mecânicas de "fiberboards" obtidos por meio da ação do sistema Fenton/CFRF na adesão de fibras de polpa termomecânica. Foi testada a reação entre $\mathrm{Fe}^{3+}\left(\right.$ ou $\left.\mathrm{Fe}^{2+}\right), \mathrm{H}_{2} \mathrm{O}_{2}$ e ácido 2,3-DHB em pH 4,5 com as fibras umedecidas, seguida de prensagem. $\mathrm{O}$ tratamento com $\mathrm{Fe}^{3+}$, $\mathrm{H}_{2} \mathrm{O}_{2}$ e ácido 2,3-DHB por $60 \mathrm{~min}$ foi o que proporcionou a mais alta resistência das ligações internas das fibras no processo úmido, 0,689 Pa. O tratamento com $1 \times 10^{-2} \mathrm{~mol} \mathrm{~L}^{-1} \mathrm{de} \mathrm{Fe}^{2+}$ e $2 \times 10^{-2} \mathrm{~mol}$ $\mathrm{L}^{-1}$ de $\mathrm{H}_{2} \mathrm{O}_{2}$ por apenas 5 min também proporcionou alta resistência, 0,502 Pa. O uso de $\mathrm{H}_{2} \mathrm{O}_{2}$ sem nenhum aditivo não foi efetivo. Reações prolongadas (180 min) causaram redução de resistência para todos as amostras. Em um outro conjunto de análises, foi avaliada a ação dos mesmos compostos em pH 4,5, seguido de prensagem com menor teor de umidade das polpas. Vários controles (sem a suplementação de ácido 2,3-DHB, $\mathrm{FeCl}_{3}$ ou $\mathrm{H}_{2} \mathrm{O}_{2}$ ) foram desenvolvidos para o processo a seco. Foi observado que apenas $1 \times 10^{-2} \mathrm{~mol} \mathrm{~L}^{-1}$ de $\mathrm{FeCl}_{3}$ e $9,3 \%(\mathrm{~m} / \mathrm{m})$ de $\mathrm{H}_{2} \mathrm{O}_{2}$ foi suficiente para possibilitar alta resistência das ligações entre fibras e módulos de ruptura e elasticidade satisfatórias, sugerindo que a lignina e extrativos presentes na superfície externa atuaram de forma semelhante ao $\mathrm{CFRF}$, dispensando seu uso. $\mathrm{O}$ tratamento com alta concentração de CFRF (0,2 mol L-1), $\mathrm{FeCl}_{3}\left(2 \times 10^{-3} \mathrm{~mol} \mathrm{~L}^{-1}\right)$ e $\mathrm{H}_{2} \mathrm{O}_{2}$ $(9,3 \% \mathrm{~m} / \mathrm{m})$ gerou o "fiberboard" com maior resistência de ligações internas $(0,576 \mathrm{~Pa})$, inclusive quando comparado ao "fiberboard" produzido com resina uréia-formaldeído (0,447 Pa, método convencional). No entanto, os módulos de ruptura e elasticidade apresentaram valores bem menores em relação às outras amostras.

\section{CONCLUSÕES}

Este trabalho evidencia os benefícios da adição de CFRF para assistir a reação de Fenton na degradação de xenobióticos por $\mathrm{H}_{2} \mathrm{O}_{2}$ e um íon metálico como $\mathrm{Fe}^{3+}$ ou $\mathrm{Fe}^{2+}$. A adição desses compostos dispensa o requerimento de meio reacional muito ácido ( $\mathrm{pH}$ entre 2 e 3) em reações do tipo Fenton, pois formam complexos solúveis com os íons metálicos em valores de pH entre 5 e 6,5. A aplicação 
do sistema Fenton/CFRF na destruição de um determinado xenobiótico depende da seleção de um CFRF apropriado para cada caso e da otimização das condições de reação. Cabe relembrar que a concentração de CFRF deve ser baixa, pois caso contrário pode haver efeito inibitório devido à reação dos radicais livres com o próprio CFRF. Esse é um aspecto favorável, pois é necessária uma quantidade mínima de CFRF nessas reações.

Além da degradação de xenobióticos, alguns estudos recentes indicam a possibilidade de aplicações do sistema Fenton/CFRF na remoção de tintas de impressão em papéis e na geração de radicais fenoxila nas superfícies de fibras para obtenção de painéis ("fiberboards").

Um trabalho interessante que deveria ser realizado consiste em estudar a eficiência do sistema Fenton/CFRF em comparação com outros POA em amostras reais e, principalmente, avaliar a viabilidade econômica destes processos. Embora os trabalhos descritos nessa revisão utilizem reagentes comerciais de grau analítico, seria ideal a obtenção destes de fontes naturais de baixo valor agregado, como a lignina e os extrativos presentes nas plantas.

\section{AGRADECIMENTOS}

À CAPES, ao CNPq, à FAPESP e SCTDE/SP. A. Aguiar agradece especialmente à FAPESP, processo 03/04465-6 e J. Rodríguez agradece à FONDECYT, projeto 1040619.

\section{REFERÊNCIAS}

1. Gogate, P.; Pandit, A.; Adv. Environ. Res. 2004, 8, 553.

2. Halliwell, B. Em Wood Deterioration and Preservation - Advances in our Changing World; Goodell, B.; Nicholas, D. D.; Schultz, T. P., eds.; ACS Symp. Ser 845, American Chemical Society: Washington, 2003, cap. 2.

3. Reading, N. S.; Welch, K. D.; Aust, S. D. Em ref. 2, cap. 3.

4. Wardman, P.; Candeias, L. P.; Radiat. Res. 1996, 145, 523.

5. Walling, C.; Acc. Chem. Res. 1998, 31, 155.

6. Hammel, K. E.; Kapich, A. N.; Jensen Jr., K. A.; Ryan, Z. C.; Enzyme Microb. Technol. 2002, 30, 445.

7. Liu, R.; Goodell, B.; Jellison, J.; Amirbahman, A.; Environ. Sci. Technol. 2005, 39, 175.

8. Bielski, B. H. J.; Cabelli, D. E.; Arudi, R. L.; Ross, A. B.; J. Phys. Chem. Ref. Data 1985, 14, 1041.

9. Schlosser, D.; Fahr, K.; Karl, W.; Wetzstein, H.; Appl. Environ. Microbiol. 2000, 66, 2479.

10. Fukushima, M.; Tatsumi, K.; Environ. Sci. Technol. 2001, 35, 1771.

11. Kramer, C.; Kreisel, G.; Fahr, K.; Kassbohrer, J.; Schlosser, D.; Appl. Microb. Biotechnol. 2004, 64, 387.

12. Sun, Y.; Pignatello, J.; J. Agric. Food Chem. 1992, 40, 322.

13. Sun, Y.; Pignatello, J.; J. Agric. Food Chem. 1993, 41, 308.

14. Li, Z. M.; Shea, P. J.; Comfort, S. D.; Chemosphere 1998, 36, 1849.

15. Fukushima, M.; Tatsumi, K.; Morimoto, K.; Environ. Sci. Technol. 2000, 34, 2006.

16. Nappi, A. J.; Vass, E.; Biochim. Biophy. Acta, Gen. Subj. 1997, 1336, 295.

17. Chen, F.; Ma, W. H.; He, J. J.; Zhao, J. C.; J. Phys. Chem. A 2002, 106, 9485 .

18. Walling, C.; Acc. Chem. Res. 1975, 8, 125.

19. Goodell, B.; Jellison, J.; Liu, J.; Daniel, G.; Paszczynsky, A.; Fekete, F.; Krishnamurthy, S.; Jun, L.; Xu, G.; J. Biotechnol. 1997, 53, 133.

20. Johnson, G. R. A.; Nazhat, N. B.; Saadallanazhat, R. A.; J. Chem. Soc. Faraday Trans. 1988, 84, 501.

21. Rodríguez, J.; Contreras, D.; Parra, C.; Freer, J.; Baeza, J.; Durán, N.; Water Sci. Technol. 1999, 40, 351.

22. Rodríguez, J.; Parra, C.; Contreras, D.; Freer, J.; Baeza, J.; Water Sci. Technol. 2001, 44, 251.

23. Strlic, M.; Radovic, T.; Kolar, J.; Pihlar, B.; J. Agric. Food Chem. 2002, 50,6313 .

24. Qian, Y.; Goodell, B.; Jellison, J.; The International Research Group on Wood Preservation Series, Ljubljana, Slovenia, IRG/WP 04-50223-report, 2004.
25. Qian, Y.; Goodell, B.; Jellison, J.; Felix, C. C.; J. Polym. Environ. 2004, $12,147$.

26. Mentasti, E.; Pelizzeti, E.; Saini, G.; J. Chem. Soc. Dalton Trans. 1973 23, 2690 .

27. Mentasti, E.; Pelizzeti, E.; Saini, G.; J. Inorg. Nucl. Chem. 1976, $38,785$.

28. Kerem, Z.; Jensen, K.A.; Hammel, K. E.; FEBS Lett. 1999, 446, 49.

29. Qian, Y. H.; Goodell, B.; Felix, C. C.; Chemosphere 2002, 48, 21.

30. Henry, W. P. Em ref. 2, cap. 10.

31. Seacat, A. M.; Kuppusamy, P.; Zweier, J. L.; Yager, J. D.; Arch. Biochem. Biophys. 1997, 347, 45; Kamau, P.; Jordan, R. B.; Inorg. Chem. 2002, 41, 3076.

32. Chen, R.; Pignatello, J.; Environ. Sci. Technol. 1997, 31, 2399

33. Xu, J.; Jordan, R. B.; Inorg. Chem. 1988, 27, 4563.

34. Pracht, J.; Boenigk, J.; Isenbeck-Schoter, M.; Keppler, F.; Scholer, H. F.; Chemosphere 2001, 44, 613.

35. Aguiar, A.; Ferraz, A.; Proceedings of $5^{\text {th }}$ International Symposium on Natural Polymers and Composites/ $8^{\text {th }}$ Brazilian Symposium on the Chemistry of Lignins and other Wood Components, São Pedro, Brasil, 2004.

36. Aguiar, A.; Souza-Cruz, P.; Ferraz, A.; Enzyme Microb. Technol. 2006, 38 , 873; Moran, J. F.; Klucas, R. V.; Grayer, R. J.; Abian, J.; Becana, M.; Free Radical Biol. Med. 1997, 22, 861; Briante, R.; Febbraio, F.; Nucci, R.; J. Agric. Food Chem. 2003, 51, 6975.

37. Hamilton, G. A.; Hanifin, J. W.; Friedman, J. P.; J. Am. Chem. Soc. 1966 , 88,5269

38. Nam, K.; Rodríguez, W.; Kukor, J.; Chemosphere 2001, 45, 11.

39. Chen, F.; He, J. J.; Zhao, J. C.; Yu, J. C.; New J. Chem. 2002, 26, 336.

40. Oviedo, C.; Contreras, D.; Freer, J.; Rodríguez, J.; Fresenius Environ. Bull. 2003, 12, 1323.

41. Oviedo, C.; Contreras, D.; Freer, J.; Rodríguez, J.; Environ. Technol. 2004, 25, 801 .

42. Pignatello, J. J.; Baehr, K.; J. Environ. Qual. 1994, 23, 365.

43. Mai, C.; Majcherczyk, A.; Schormann, W.; Hüttermann, A.; Polym. Degrad. Stab. 2002, 75, 107

44. Nagarathnamma, R.; Bajpai, P.; Bajpai, P. K.; Process Biochem.1999, 34, 939.

45. Nerud, F.; Baldrian, P.; Gabriel, J.; Ogbeifun, D.; Chemosphere 2001, 44, 957; Shah, V.; Verma, P.; Stopka, P.; Gabriel, J.; Baldrian, P.; Nerud, F.; Appl. Catal., B 2003, 46, 287; Verma, P.; Baldrian, P.; Nerud, F.; Chemosphere 2003, 50, 975; Verma, P.; Baldrian, P.; Gabriel, J.; Trnka, T.; Nerud, F.; Chemosphere 2004, 57, 1207; Verma, P.; Shah, V.; Baldrian, P.; Gabriel, J.; Stopka, P.; Trnka, T.; Nerud, F.; Chemosphere 2004, 54, 291; Gabriel, J.; Baldrian, P.; Verma, P.; Cajthaml, T.; Merhautová, V.; Eichlerová, I.; Stoytchev, I.; Trnka, T.; Stopka, P.; Nerud, F.; Appl. Catal., B 2004, 51, 159.

46. Tuomela, M.; Vikman, M.; Hatakka, A.; Itavaara, M.; Bioresour. Technol. 2000, 72, 169

47. Paciolla, M. D.; Davies, G.; Jansen, S. A.; Environ. Sci. Technol. 1999, 33 , 1814.

48. Voelker, B.; Sulzberger, B.; Environ. Sci. Technol. 1996, 30, 1106.

49. Lindsey, M. E.; Tarr, M. A.; Chemosphere 2000, 41, 409.

50. Lindsey, M. E.; Tarr, M. A.; Water Res. 2000, 34, 2385.

51. Beltran, F. J.; González, M.; Rivas, F. J.; Alvarez, P.; Water, Air, Soil Pollut. 1998, 105, 685 .

52. Vione, D.; Merlo, F.; Maurino, V.; Minero, C.; Environ. Chem. Lett. 2004, 2, 129.

53. Ferraz, A. Em Fungos, uma introdução à biologia, bioquímica $e$ biotecnologia; Esposito, E.; Azevedo, J. L., eds.; Educs: Caxias do Sul, 2004, cap. 6.

54. Koenigs, J. W.; Wood Fiber 1974, 6, 66.

55. Shimada, M.; Akamtsu, Y.; Tokimatsu, T.; Mii, K.; Hattori, T.; J. Biotechnol. 1997, 53, 103

56. Paszczynski, A.; Crawford, R.; Funk, D.; Goodell, B.; Appl. Environ Microbiol. 1999, 65, 674

57. Jensen, K. A.; Ryan, Z. C.; Wymelenberg, A. V.; Cullen, D.; Hammel, K. E.; Appl. Environ. Microb. 2002, 68, 2699.

58. Goodell, B.; Jellison, J.; Liu, J.; Krishnamurthy, S.; US pat. 6,046,375 2000 (CA 2000:220739).

59. Enoki, A.; Tanaka, H.; Itakura, S. Em ref. 2, cap. 8.

60. Qian, Y.; Goodell, B.; Bioresour. Technol. 2005, 96, 913.

61. Parra, C.; Oses, R.; Ruiz, J.; Urtubia, I.; Baeza, J.; Freer, J.; Rodriguez, J.; Proceedings of XII International Conference on Biotechnology in the Pulp and Paper Industry, Vancouver, Canadá, 1998.

62. Yelle, D.; Goodell, B.; Gardner, D. J.; Amirbahman, A.; Winistorfer, P.; Shaler, S.; Forest Prod. J. 2004, 54, 73. 